\title{
Concurrent mapping of multiple epigenetic marks and co-occupancy using ACT2-seq
}

\author{
Benjamin Carter ${ }^{\dagger}$, Wai Lim Kư ${ }^{\dagger}$, Joe Pelt and Keji Zhao*
}

\begin{abstract}
Background: Genome-wide profiling of epigenetic marks is a core technology in molecular genetics. Co-occupancy of different epigenetic marks or protein factors at the same genomic locations must often be inferred from multiple independently collected data sets. However, this strategy does not provide direct evidence of co-enrichment in the same cells due to the existence of cellular heterogeneity. To address this issue, we have developed a technique termed ACT2-seq that is capable of concurrently profiling multiple epigenetic marks in a single biological sample. In addition to reducing the numbers of samples required for experiments, ACT2-seq is capable of mapping co-occupancy of epigenetic factors on chromatin. This strategy provides direct evidence of co-enrichment without requiring complex single-molecule, single-cell, or magnetic bead-based approaches.
\end{abstract}

Results: We concurrently profiled pairs of two epigenetic marks using ACT2-seq as well as three marks in individual samples. Data obtained using ACT2-seq were found to be reproducible and robust. ACT2-seq was capable of cleanly partitioning concurrently mapped data sets that exhibited distinct enrichment patterns. Using ACT2-seq, we identified distinct relationships between co-occupancy of specific histone modifications and gene expression patterns.

Conclusions: We conclude that ACT2-seq presents an attractive option for epigenomic profiling due to its ease of use, potential for reducing sample and sequencing costs, and ability to simultaneously profile co-occupancy of multiple histone marks and/or chromatin-associated proteins.

Keywords: Co-occupancy, Co-enrichment, ACT-seq, Tagmentation, Epigenetic marks

\section{Background}

Cellular processes such as differentiation and responses to environmental stimuli often involve persistent changes in gene expression. In eukaryotic cells, these processes are facilitated by chromatin-based features including covalent histone modifications, chromatin accessibility, and DNA methylation. Such features are referred to as "epigenetic marks" and are deposited and recognized by chromatin-associated "writers" and "readers" [1-3]. In addition to binding chromatin-associated machinery, epigenetic marks can influence gene expression through

*Correspondence: zhaok@nhlbi.nih.gov

†Benjamin Carter and Wai Lim Ku contributed equally to this work Laboratory of Epigenome Biology, Systems Biology Center, National Heart, Lung and Blood Institute, NIH, Bethesda, MD 20892, USA other mechanisms including modifying the mechanical properties of chromatin [4] or promoting changes in higher-order chromatin organization [5-7].

The most widely adopted method for mapping epigenetic marks has been chromatin immunoprecipitation followed by sequencing (ChIP-seq), in which antibodies that target the epigenetic mark of interest are used to precipitate and isolate chromatin fragments that bear the mark $[8,9]$. DNA is then purified from the enriched chromatin fragments and sequenced using high-throughput sequencing technologies. The resulting sequence reads are mapped to the organism's reference genome to generate a genome-wide enrichment profile for the targeted epigenetic mark. Data obtained using ChIP-seq is often robust and reproducible, making it an invaluable tool in the field of molecular genetics. original author(s) and the source, provide a link to the Creative Commons licence, and indicate if changes were made. The images or other third party material in this article are included in the article's Creative Commons licence, unless indicated otherwise in a credit line to the material. If material is not included in the article's Creative Commons licence and your intended use is not permitted by statutory regulation or exceeds the permitted use, you will need to obtain permission directly from the copyright holder. To view a copy of this licence, visit http://creativecommons.org/licenses/by/4.0/. The Creative Commons Public Domain Dedication waiver (http://creativeco mmons.org/publicdomain/zero/1.0/) applies to the data made available in this article, unless otherwise stated in a credit line to the data. 
Due to the important roles of epigenetic marks in controlling transcription and chromatin organization, it is valuable to understand which epigenetic factors cooccupy a gene promoter or other regulatory region. Identifying co-occupancy of chromatin features, such as the binding of transcription factors along with the presence of specific histone modifications, provides important mechanistic insights into chromatin-based phenomena. For example, co-occupancy of two chromatin features at a gene regulatory region raises the possibility that they function cooperatively or synergistically and can clarify models of the mechanisms of gene regulation. Co-occupancy of epigenetic marks and/or chromatin-binding proteins is often inferred using their enrichment at the same genomic locations from independent epigenomic datasets $[10,11]$. However, most available methods for profiling epigenomic marks provide a single aggregate signal compiled from thousands or millions of cells. Enrichment profiles generated from such samples are not sufficient to establish that co-occupancy occurs due to the extensive effects of cell-to-cell heterogeneity in epigenetic states, which is present even among highly similar and clonally derived cells [12]. Thus, genomic regions exhibiting enrichment for multiple epigenetic marks in different samples can arise from heterogeneous subpopulations of cells in which the two marks are seldom or never present at the same time in the same cells [12].

We previously developed a chromatin profiling method, antibody-guided chromatin tagmentation sequencing (ACT-seq), based on the DNA transposition activity of $\operatorname{Tn} 5$ transposase [13]. In ACT-seq, $\operatorname{Tn} 5$ is fused to Protein A to facilitate antibody-based binding of the transposase to targeted epigenetic marks and transcription factors. This allows chromatin fragmentation and adapter ligation to be performed by the Tn 5 enzyme during a single experimental step specifically at genomic locations enriched for the epigenetic mark of interest. ACT-seq dramatically reduces the need for experimental optimization relative to ChIP-seq and its derivative methods. In this study, we present an expanded and streamlined version of ACT-seq known as antibodyguided chromatin tagmentation for two factors followed by sequencing (ACT2-seq, ACT2). Like ACT-seq, ACT2seq can be completed in one day of bench work and does not require expensive reagents such as magnetic beads, sonicators, or library preparation kits. ACT2-seq makes use of barcoded transposase adapters to enable concurrent profiling of two epigenetic marks in a single biological sample, which can reduce the numbers of samples required for experiments. Importantly, this barcoding strategy simultaneously probes for co-enrichment of epigenetic marks, which previously required technically challenging or laborious approaches involving single cells, single-molecule fluorescence, or sequential ChIP $[14,15]$. This approach provides direct evidence of cooccupancy of epigenetic marks, avoiding the issue of cellular heterogeneity that confounds interpretation of co-enrichment using bulk-cell ChIP-seq data. We conclude that these features make ACT2 an appealing option for whole-genome profiling of epigenetic factors.

\section{Results}

ACT2 uses a fusion of Protein A with Tn5 transposase (PA-Tn5), which was previously introduced for use with ACT-seq [13]. With ACT2, however, the oligonucleotide transposase adapters are barcoded to enable different antibodies to be used in the same biological sample (Fig. 1A, top). Separate aliquots of PA-Tn5 enzyme are bound to discrete combinations of adapters and antibodies, which allows for concurrent binding of multiple labeled PA-Tn5 complexes to chromatin (Fig. 1A, bottom). After the binding steps, any unbound PA-Tn 5 complexes are washed away, and tagmentation is initiated to capture signals specifically arising from bound genomic locations. This strategy enables the DNA fragments generated by ACT2 to be sorted into separate signals based on the adapter barcodes present on the fragment.

To examine whether data obtained using the modified ACT2 adapters were robust and reproducible, we probed multiple epigenetic factors in cultured human GM12878 lymphoblast cells: trimethylation of lysine 4 on histone H3 (H3K4me3), acetylation of lysine 27 on histone H3 (H3K27ac), trimethylation of lysing 9 on histone $\mathrm{H} 3$ (H3K9me3), and RNA Polymerase II (RNA Pol II). These factors were selected to profile both transcriptionally repressed and active genomic regions as well as a chromatin-associated protein complex. Immunoglobulin G (IgG) was included as a control for non-specific binding and enzyme activity. Visual inspection of the peak patterns produced by ACT2 at genomic regions enriched for the "active" chromatin marks revealed a high level of similarity to ENCODE-validated ChIP-seq data sets (Fig. 1B). In comparison, the IgG-bound complex produced sparse and intermittent signals with little to no peak formation, indicating that the enrichment patterns detected using ACT2 arise from specific antibody binding to target sites. We similarly examined genomic regions enriched for H3K9me3 and observed strong similarities between the enrichment patterns obtained using ACT2 and those from the ENCODE ChIP-seq data (Fig. 1C). To further examine the robustness of data obtained using ACT2, we computationally identified regions of enrichment ("peaks") for each biological replicate of H3K4me3, $\mathrm{H} 3 \mathrm{~K} 27 \mathrm{ac}$, and H3K9me3. The read densities within these peaks were found to be highly similar between biological 


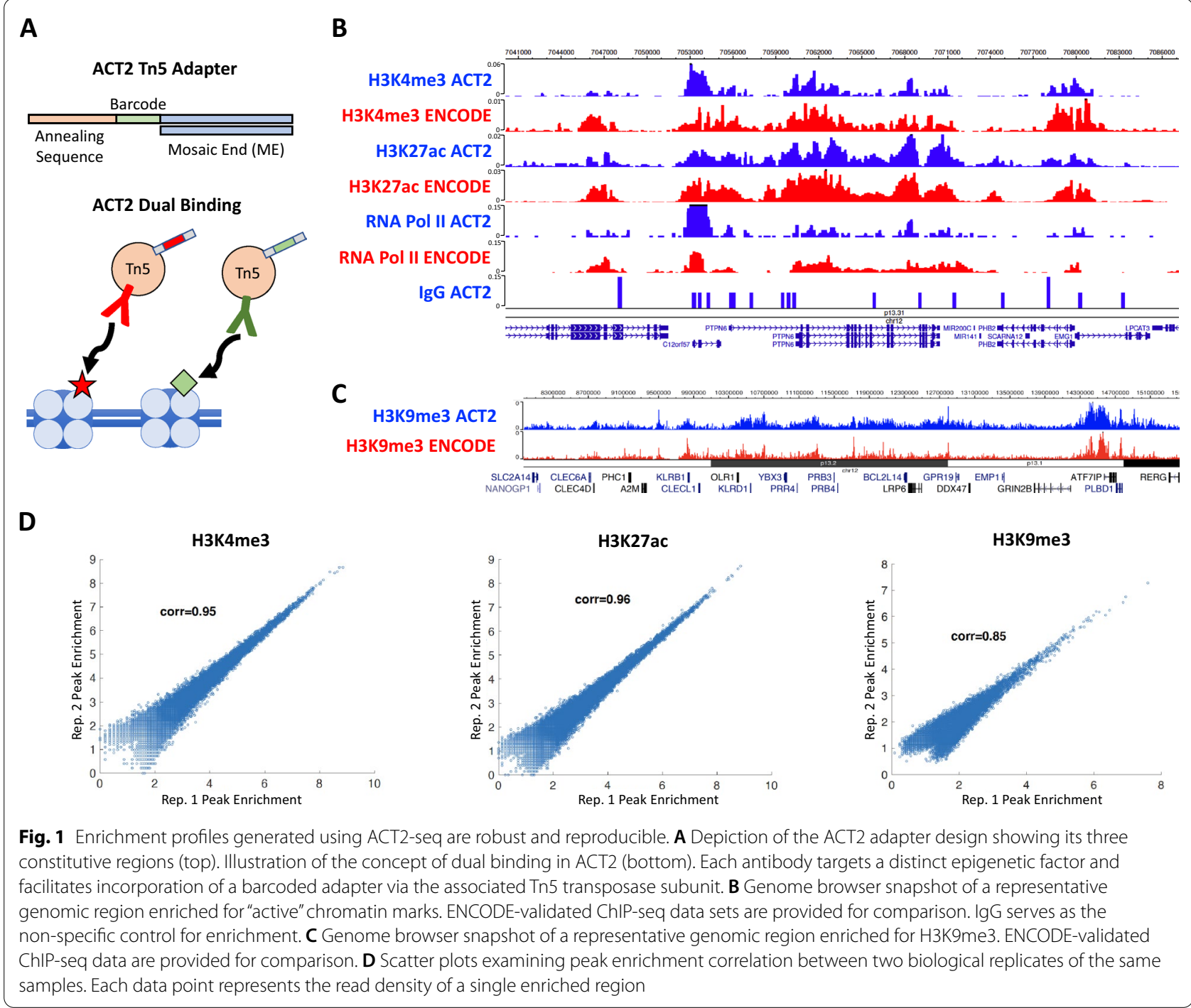

replicates of the same treatment: over $85 \%$ correlation in each case (Fig. 1D).

The ACT2 barcoding strategy (Fig. 1A) enables concurrent profiling of multiple epigenetic marks in a single biological sample. To investigate whether ACT2 is capable of efficiently partitioning the multiple data sets generated from a single sample, we examined enrichment patterns for epigenetic factors that were mapped concurrently. Many previous studies have identified distinct enrichment signatures for specific "active" epigenetic marks at transcription start sites (TSSs) and enhancers linked to highly expressed genes [16-18]. Consistent with these studies, we observed strong enrichment of both H3K4me3 and RNA Pol II at gene TSSs when both marks were mapped concurrently in the same ACT2 sample (Fig. 2A, left). In contrast, co-profiling of H3K4me3 alongside the repressive histone modification $\mathrm{H} 3 \mathrm{~K} 9 \mathrm{me} 3$ resulted in strikingly different patterns of average enrichment (Fig. 2A, middle). Further, we found that the distinct H3K9me3 enrichment profile was maintained even when this mark was co-profiled alongside two active histone modifications in the same sample (Fig. 2A, right). These results indicate that ACT2 is capable of partitioning data sets that exhibit disparate enrichment patterns from the same biological sample.

To further examine whether ACT2 efficiently resolves co-profiled data sets, we visualized enrichment of these factors across different classes of genes and genomic elements. Using publicly available RNA-seq data from the same cell type [19], we identified gene sets corresponding to the top and bottom quartiles of expression. We examined average enrichment of three co-profiled 


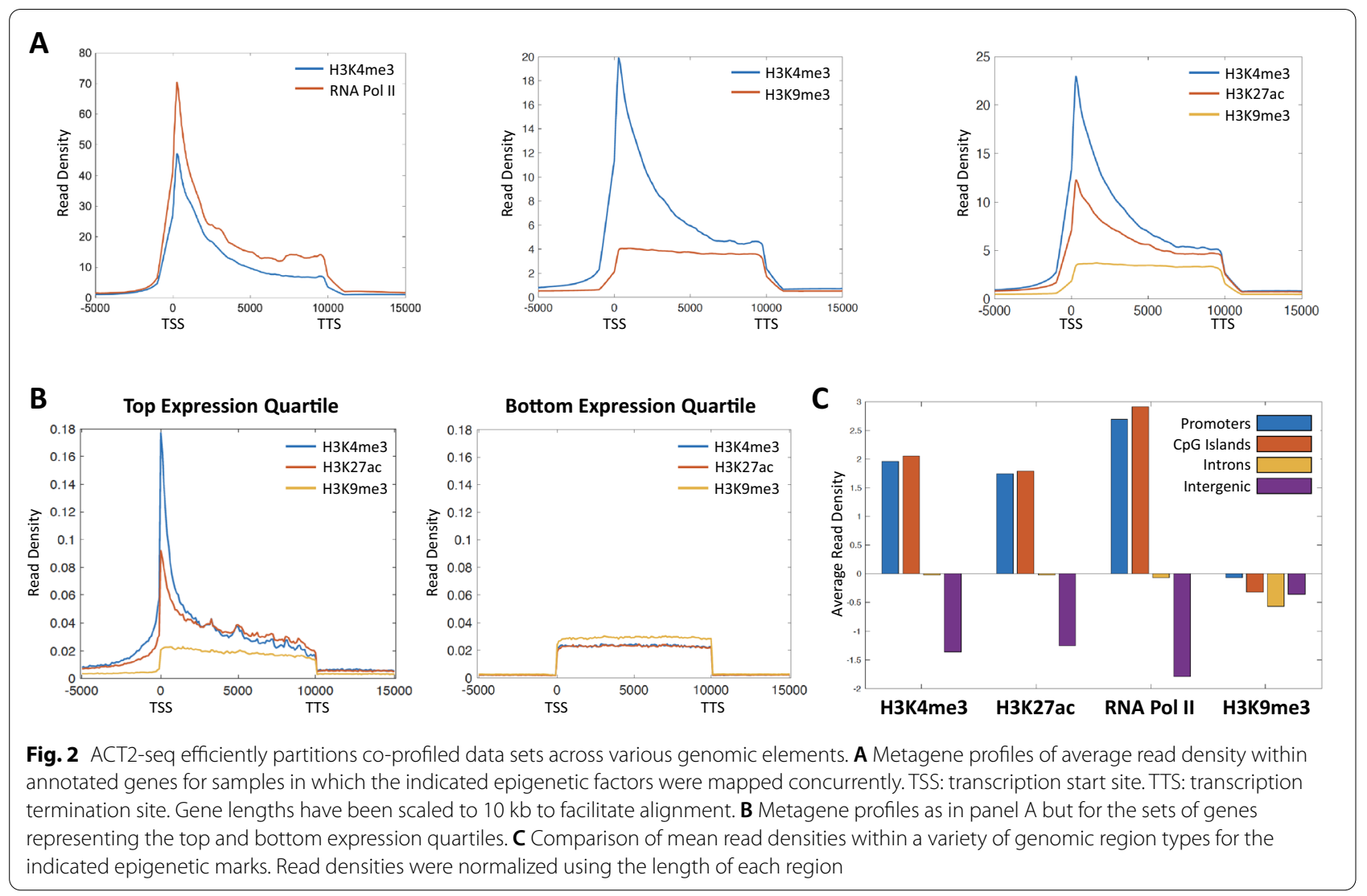

epigenetic marks (Fig. 2A, right) at these categories of genes and observed pronounced enrichment of the active histone marks at active TSSs without concurrent H3K9me3 enrichment (Fig. 2B, left). In contrast, genes in the bottom quartile of expression were not enriched for the active marks at the TSSs, suggesting an expression-dependent role for these factors. Based on these results, we predicted that $\mathrm{H} 3 \mathrm{~K} 9 \mathrm{me} 3$ would exhibit distinct enrichment patterns relative to the active marks across other classes of genomic elements as well. To test this prediction, we calculated relative enrichment across a variety of genomic elements including promoters, CpG islands, introns, and intergenic regions. For the active epigenetic marks H3K4me3, H3K27ac, and RNA Pol II, we observed elevated enrichment at gene promoters and at $\mathrm{CpG}$ islands, which are often associated with proximal gene promoters in humans [17, 20] (Fig. 2C). Consistent with our conjecture, we did not observe enrichment of H3K9me3 at these elements, which supports the distinct distribution of H3K9me3 relative to the active marks. For comparison, all of the epigenetic marks we examined were relatively sparsely enriched at introns and intergenic regions. Taken together, these analyses support the robustness of ACT2 co-profiling for epigenetic marks with distinct enrichment patterns at various classes of genes and genomic elements.

In addition to concurrently mapping multiple epigenetic factors, ACT2 simultaneously probes co-occupancy of the factors across the genome. This capability arises from the use of oligonucleotide adapters with distinct barcodes for each enzyme-antibody complex. For example, when using two antibodies " $\mathrm{A}$ " and "B", three distinct configurations of barcodes could be present at either end of the sequencing reads when the two factors are in close spatial proximity on chromatin (Fig. 3A). A DNA fragment with both barcodes matching the enzyme-antibody complex associated with either antibody A or B indicates a signal arising from that antibody alone. However, fragments bearing an antibody. A barcode at one end and an antibody $\mathrm{B}$ barcode at the other end represent a signal arising from both antibodies simultaneously. Importantly, these barcode-mismatched fragments can only arise when both epigenetic factors are present together in close proximity and thus directly indicate co-occupancy of the two factors on the same chromatin strand. Thus, ACT2 data files can be partitioned into sub-samples representing various types of enrichment or co-enrichment signals based on the barcodes of the sequence reads (see Table 1 in Methods). 


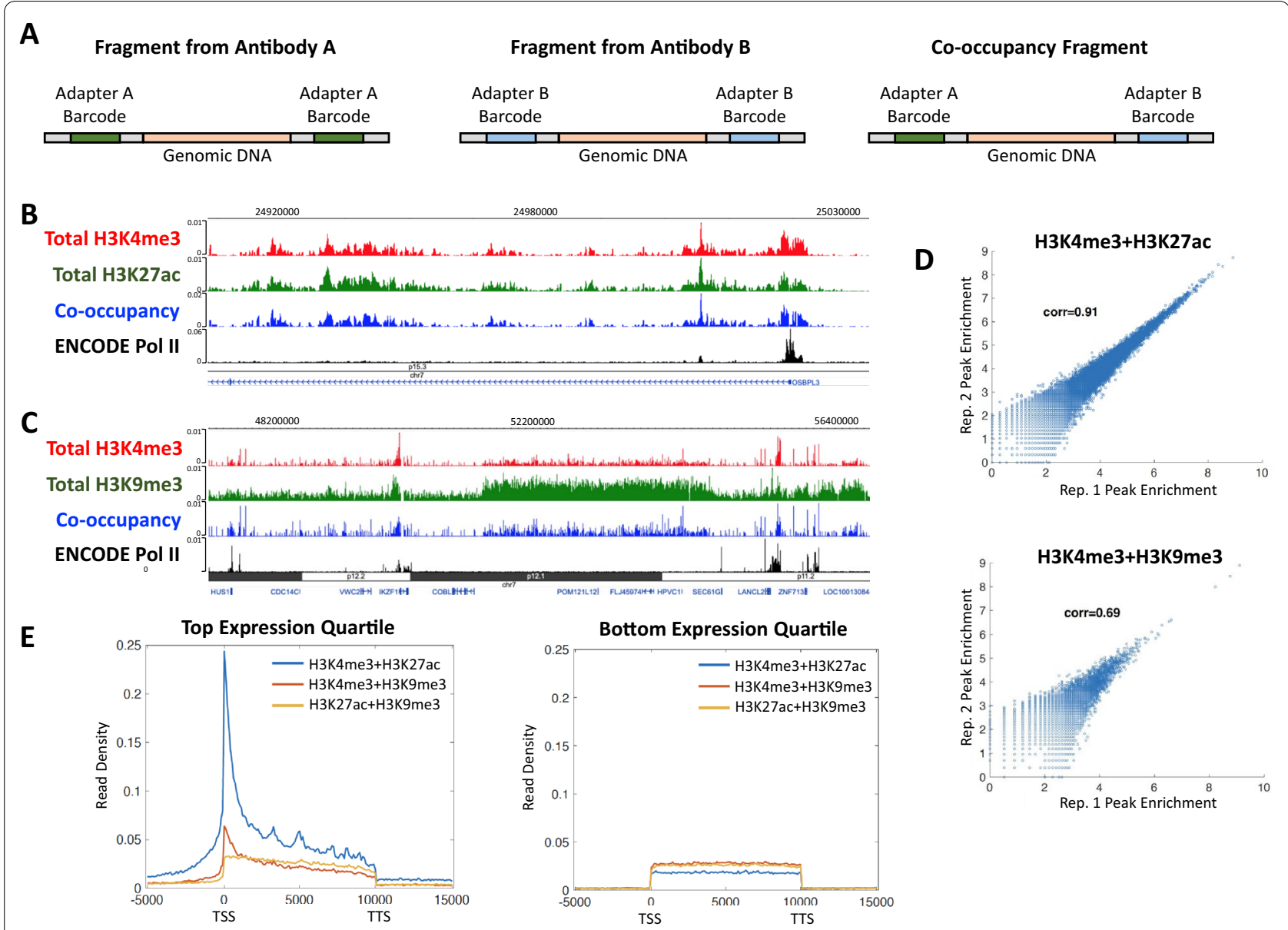

Fig. 3 ACT2-seq directly profiles co-occupancy between pairs of epigenetic factors. A Illustration of the types of DNA fragments generated by the ACT2 method. Each fragment type provides enrichment information for either antibody A, antibody B, or both (co-occupancy). B Genome browser snapshot of a representative genomic region enriched for "active" chromatin marks. The top three tracks were obtained from a single sample in which H3K4me3 and H3K27ac were mapped concurrently. Four data tracks are provided: one for total H3K4me3 signal, one for total H3K27ac signal, one for only co-occupancy reads, and one for ENCODE ChIP-Seq of RNA Pol II for comparison. C Genome browser snapshot of a representative genomic region enriched for both $\mathrm{H} 3 \mathrm{~K} 9 \mathrm{me} 3$ and $\mathrm{H} 3 \mathrm{~K} 4 \mathrm{me} 3$. The top three tracks were obtained from a single sample in which H3K4me3 and H3K9me3 were mapped concurrently. Four data tracks are provided: one for total H3K4me3 signal, one for total H3K9me3 signal, one for only co-occupancy reads, and one for ENCODE ChIP-seq of RNA Pol II for comparison. Note the region of high H3K9me3 enrichment that roughly correlates to chromosome band p12.1 in black. D Scatter plots examining peak enrichment correlation between two biological replicates of co-enrichment data. Each data point represents the read density of a single enriched region. E Metagene profiles of average read density within annotated genes for co-enrichment of the indicated epigenetic factors. All three co-enrichment data sets were obtained from a single concurrent mapping of H3K4me3, H3K27ac, and H3K9me3. TSS: transcription start site. TTS: transcription termination site. Gene lengths have been scaled to $10 \mathrm{~kb}$ to facilitate alignment. Plots are provided for gene sets representing the top and bottom expression quartiles

Table 1 Data sets partitioned from an example ACT-seq sample using two antibodies

\begin{tabular}{llll}
\hline Name & Barcode 1 & Barcode 2 & Data type \\
\hline A-only & Antibody A & Antibody A & Signal arising from antibody A and no others \\
A-total & Antibody A & Any & Total signal from all antibody A fragments \\
B-only & Antibody B & Antibody B & Signal arising from antibody B and no others \\
B-total & Antibody B & Any & Total signal from all antibody B fragments \\
Co-oc. & Antibody A & Antibody B & Signal for co-occupancy of the two factors
\end{tabular}

${ }^{a}$ Note that samples in which more than two factors are mapped concurrently will also generate additional co-enrichment data sets for each distinct combination 
To investigate the ability of ACT2 to probe co-occupancy of epigenetic factors, we used the barcode strategy discussed above to partition samples in which H3K4me3 was concurrently profiled alongside H3K27ac, H3K9me3, or both. Consistent with our previous analyses (Fig. 2), we found that co-occupancy of the active histone modifications H3K4me3 and H3K27ac resembled the shared peak patterns of both marks (Fig. 3B). In contrast, we observed many broad regions of H3K9me3 enrichment that exhibited relatively lower levels of H3K4me3. These likely represent transcriptionally repressed and/or heterochromatic chromosome domains as suggested by the overlap between the broad region of H3K9me3 enrichment and the chromosome band p12.1 found in the central region of Fig. 3C. Visual inspection of the data revealed that such $\mathrm{H} 3 \mathrm{~K} 9 \mathrm{me} 3$-rich domains also exhibited relatively lower levels of H3K4me3+H3K9me3 cooccupancy compared to most isolated peaks (Fig. 3C). To determine whether co-occupancy signals obtained using ACT2 were reproducible, we compared peak enrichment levels between biological replicates as was done in Fig. 1D. We observed very high reproducibility in peak enrichment for replicates of H3K4me3+H3K27ac co-occupancy (Fig. 3D, top). The reproducibility of H3K4me3 + H3K9me3 co-occupancy was lower (Fig. 3D, bottom), likely due in part to the dissimilar enrichment profiles of these marks (Fig. 2A-C).

It should be noted that profiling more than two epigenetic factors in a single ACT2 sample will produce a separate co-occupancy data set for each pairwise combination of factors. For example, the sample in which H3K4me3, H3K27ac, and H3K9me3 were all probed concurrently (Fig. 2A, right) provided three co-occupancy data sets: H3K4me3+H3K27ac, H3K4me3 + H3K9me3, and $\mathrm{H} 3 \mathrm{~K} 27 \mathrm{ac}+\mathrm{H} 3 \mathrm{~K} 9 \mathrm{me} 3$. We constructed metagene profiles for these co-occupancy data sets at highly expressed and at lowly expressed genes (Fig. 3E). At highly expressed genes, we observed that H3K4me3 and H3K27ac exhibited elevated average levels of cooccupancy at TSSs, whereas co-occupancy of either of these marks with $\mathrm{H} 3 \mathrm{~K} 9 \mathrm{me} 3$ was present at much lower levels (Fig. 3E, left). Interestingly, H3K27ac + H3K9me3 co-occupancy exhibited a distinguishable pattern from $\mathrm{H} 3 \mathrm{~K} 4 \mathrm{me} 3+\mathrm{H} 3 \mathrm{~K} 9 \mathrm{me}$, suggesting the presence of different mechanisms of interaction between these pairs of epigenetic marks. In contrast, lowly expressed genes did not exhibit peaks of H3K4me3 + H3K27ac co-occupancy (Fig. 3E, right), consistent with our previous observation of expression-level-dependent distributions of these marks (Fig. 2B).

The observed co-occupancy profiles among H3K27ac, H3K4me3, and H3K9me3 at high vs. low expression genes (Fig. 3E) prompted us to examine if the presence of co-occupancy among these factors at promoter or enhancer elements was predictive of gene expression levels. Genes were separated into categories based on whether the promoter was enriched for H3K4me3 alone, H3K27ac alone, or co-occupied by both histone marks. We then compared the distributions of expression for genes in each category. We observed that co-occupancy of H3K4me3 and H3K27ac at gene promoters was correlated with a statistically significant elevation in gene expression compared to promoters enriched for either mark alone (Fig. 4A). However, the expression profile for promoters enriched for H3K4me3 alone was not significantly different from those enriched for H3K27ac alone, indicating that the expression effects arising from the presence of either mark individually are not broadly distinguishable. We performed a similar analysis of gene-proximal enhancer regions. Proximal enhancers were identified as regions enriched for H3K27ac using a distance cutoff of five kilobases from the nearest gene.

\footnotetext{
(See figure on next page.)

Fig. 4 Co-enriched genomic elements identified using ACT2 exhibit distinct gene expression profiles. A-D Violin plots depicting expression profiles of gene sets in the indicated categories. $p$-values were obtained using Wilcoxon rank sum tests. A Violin plot depicting the expression profiles of genes that exhibited (co-)enrichment of H3K4me3 and/or H3K27ac at the gene promoter. Gene expression was calculated as reads per kilobase mapped (RPKM). Using a significance threshold of $a<0.005$, the null hypothesis of no difference in the gene expression profile of co-occupied promoters was rejected. B Violin plot as in panel A but using enrichment status at gene enhancers. Enhancers were defined as regions enriched for H3K27ac, and thus the H3K4me3-alone category was not included. Gene expression values were matched to enhancers based on proximity. Using a significance threshold of $a<0.005$, the null hypothesis of no difference in the gene expression profile of co-occupied enhancers was not rejected. $\mathbf{C}$ Violin plot depicting the expression profiles of genes that exhibited (co-)enrichment of H3K4me3 and/or H3K9me3 at the gene promoter. Gene expression was calculated as reads per kilobase mapped (RPKM). Using a significance threshold of a $<0.005$, the null hypothesis of no difference in the gene expression profile of co-occupied promoters was rejected. D Violin plot as in panel C but using ENCODE ChIP-seq data. Since co-occupancy cannot be examined in these data, the presence of overlapping $\mathrm{H} 3 \mathrm{~K} 4 \mathrm{me} 3$ and $\mathrm{H} 3 \mathrm{~K} 9 \mathrm{me} 3$ peaks within the promoter regions was used as a proxy. Using a significance threshold of $a<0.005$, the null hypothesis of no difference in the gene expression profile of promoters containing overlapping expression peaks was rejected. E Scatter plot examining the correlation between $\mathrm{H} 3 \mathrm{~K} 9 \mathrm{me} 3$ and $\mathrm{H} 3 \mathrm{~K} 4 \mathrm{me} 3 \mathrm{read}$ densities across the genome. Each data point represents a genomic fragment of five kilobases in length. Fragments exhibiting only H3K9me3 enrichment with no detectable $\mathrm{H} 3 \mathrm{~K} 4 \mathrm{me} 3$ enrichment are depicted in red on the $y$-axis. $\mathbf{F}$ Violin plot depicting the expression profiles of genes that exhibited enrichment of H3K9me3 but not H3K4me3 at the transcription start site (TSS) vs. all other genes. Gene expression was calculated as reads per kilobase mapped (RPKM). Using a significance threshold of $a<0.005$, the null hypothesis of no difference in the gene expression profile of these genes vs. all other genes was rejected
} 


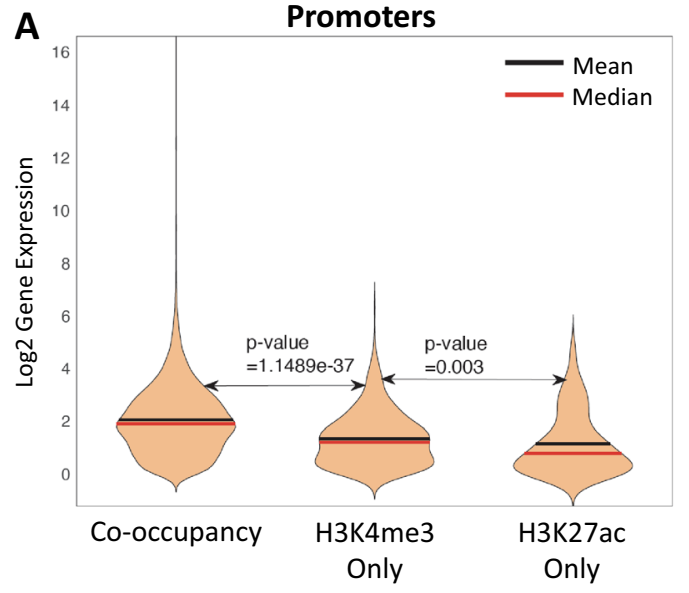

C

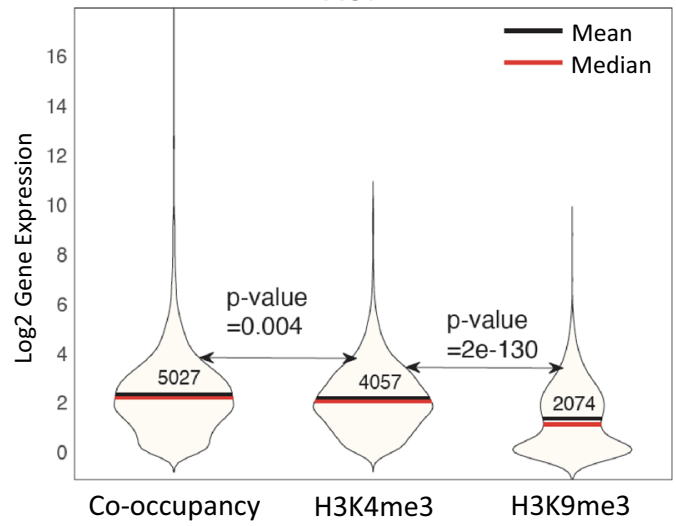

E

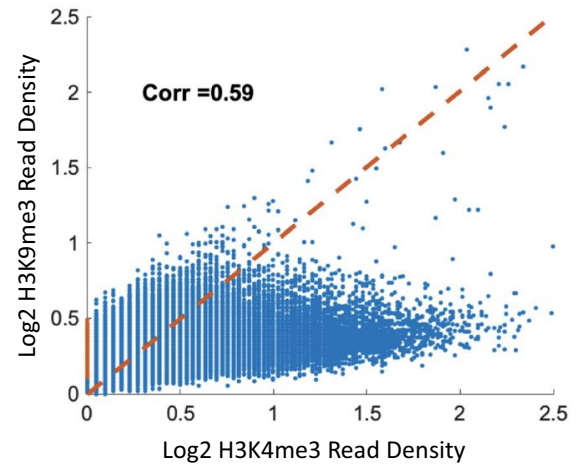

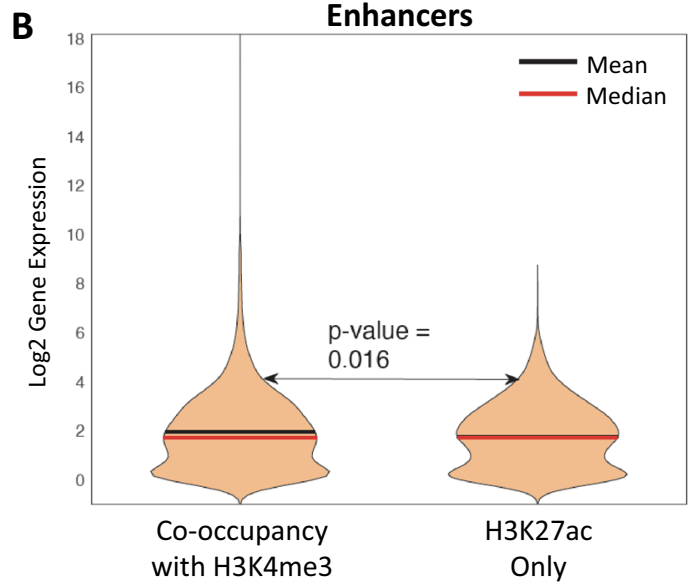

D

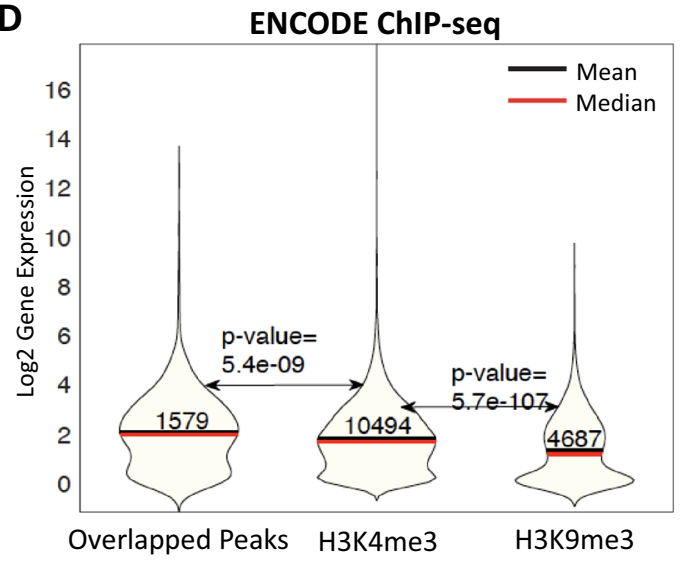

$\mathbf{F}$

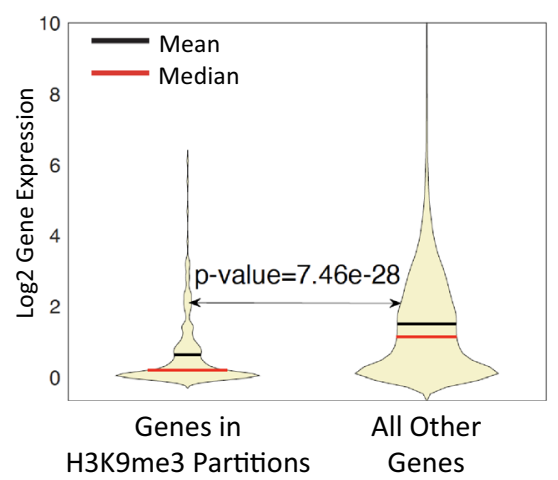

Fig. 4 (See legend on previous page.)

Proximal enhancers were sorted into two categories based on whether they were enriched for H3K27ac alone or co-occupied with H3K27ac and H3K4me3. We did not observe a significant difference in gene expression for co-occupied proximal enhancers compared to enhancers enriched for H3K27ac alone (Fig. 4B). Together, these data indicate that co-occupancy of H3K4me3 and $\mathrm{H} 3 \mathrm{~K} 27 \mathrm{ac}$ is correlated with higher gene expression levels if present at promoters, but do not broadly affect gene expression if present at proximal enhancer elements.

Interestingly, we identified a distinct class of genes that exhibited co-occupancy of H3K4me3 and $\mathrm{H} 3 \mathrm{~K} 9 \mathrm{me} 3$ at the promoter (Fig. 4C). This is notable due to the broader differences in the genomic distributions of these marks (Fig. 2A-C). While this class of co-occupied promoters is correlated with marginally elevated 
gene expression, the effect is very small compared to co-occupancy of H3K4me3+H3K27ac (Fig. 4C vs. A). Precedent for this effect can be found in ENCODE ChIP-seq data for these marks. While it is not possible to examine co-occupancy using ChIP-seq, we identified gene promoters containing overlapping H3K4me3 and H3K9me3 enrichment peaks as a proxy. Such dualenriched promoters exhibited significantly elevated expression levels compared to promoters enriched for either mark alone (Fig. 4D). Together, these data support the presence of a separate class of gene promoters exhibiting H3K4me3 and H3K9me3 co-enrichment that are associated with a marginally elevated gene expression distribution. Importantly, promoters enriched for H3K9me3 alone were associated with sharply reduced expression levels in both the ACT2 and ChIP-seq data sets (Fig. 4C-D), consistent with the characterized role of this mark in transcriptional repression. Notably, direct probing of co-occupancy revealed a higher number of co-enriched genes (Fig. 4C) compared to simple overlapping of ChIP-seq peaks (Fig. 4D), suggesting that the ACT2 approach is more sensitive.

The unexpected presence of promoter co-occupancy of H3K4me3 and H3K9me3, which are normally associated with active and repressed transcriptional states, respectively, prompted us to examine genome-wide correlations between these marks in a gene- and peak-agnostic manner. We computationally sectioned the genome into five-kilobase segments and examined the read densities of H3K4me3 and H3K9me3 within each segment (Fig. 4E). In contrast to the H3K4me3 levels that have a large dynamic range (Fig. 4E, increasing $x$-axis values), the H3K9me3 level have a relatively narrow dynamic range. We examined the expression of genes associated with relatively high levels of $\mathrm{H} 3 \mathrm{~K} 9 \mathrm{me} 3$ but with no or very low levels of H3K4me3 signals (Fig. 4E, highlighted in red (comprising 4,370 genome segments and 1,852 genes). We found that expression of the genes in these segments were sharply reduced relative to the larger gene population (Fig. 4F), again consistent with the repressive nature of $\mathrm{H} 3 \mathrm{~K} 9 \mathrm{me} 3$ and indicating that our genome partitioning is accurately capturing the roles of these histone marks. Importantly, a small fraction of genomic segments exhibited co-linear read densities for both H3K4me3 and H3K9me3 (Fig. 4E, upper-right quadrant). In other words, a subcategory of genomic segments eluded the standard trend and exhibited elevated enrichment of both H3K4me3 and H3K9me3. Combined with the unexpected presence of H3K4me3+H3K9me3 co-occupied gene promoters revealed using $\mathrm{ACT} 2$, these data suggest the existence of novel mechanisms of genomic interplay between these seemingly disparate histone modifications.

\section{Discussion}

ACT2-seq relies on tagmentation by the $\operatorname{Tn} 5$ transposase subunit of the PA-Tn5 enzyme to generate DNA fragments for sequencing. This technology greatly facilitates the speed and simplicity of the ACT2 protocol but presents some technical considerations for the user. For example, generation of sequence-able genomic fragments by tagmentation requires two nearby adapter insertion events by the transposase (one for each adapter-tagged end of the fragment). If probing an epigenetic mark that is exceedingly sparsely distributed, it could prove difficult to accumulate the insertion events required to generate a substantial number of tagged fragments for sequencing. However, ACT2 may provide an advantage in this area compared to other targeted DNA-fragmentation methods. The ability to co-profile multiple epigenetic marks in the same sample presents the opportunity to introduce a larger number of tag-insertion events compared to probing a single sparsely distributed epitope alone. This could greatly increase the likelihood of generating sequenceable fragments for such epitopes.

Co-enrichment of epigenetic factors can also be investigated using single-cell methods. However, single-cell methods are much more technically complex and require methods such as drop fluidics or split-pool barcoding. In addition, even the latest single-cell protocols suffer from sparse or missing data issues that can confound co-occupancy studies or limit their use to a small number of target sites. Even under ideal circumstances, single cells generally possess more than one copy of most genes, which makes it difficult to conclusively identify co-occupancy of epigenetic factors. A primary advantage of ACT2 is the ability to directly profile co-occupancy of factors on the same chromosome strands without the need to obtain single-cell data. In addition, the simplicity of the ACT2 protocol facilitates rapid concurrent mapping of epigenomic marks and avoids the need for expensive reagents such as library preparation kits or magnetic beads.

\section{Conclusions}

ACT2-seq was demonstrated to be capable of concurrent mapping of multiple epigenetic marks in a single sample. Further, ACT2-seq simultaneously provides co-occupancy profiles between pairs of probed marks without the need for additional experimental steps. These advantages make ACT2 an appealing alternative to currently available methods for epigenomic profiling that require such laborious procedures and/or expensive reagents. Using ACT2, we identified a strong correlation between H3K4me3 + H3K27ac co-occupancy at transcription start sites and gene expression levels. Further, we identified genomic co-occupancy of H3K4me3 and H3K9me3, 
two epigenetic marks that otherwise exhibit disparate patterns of enrichment. Based on its simplicity, technical advantages, and ability to profile co-occupancy of epigenetic factors, we conclude that ACT2-seq presents an appealing alternative to currently available methods for epigenomic profiling.

\section{Materials and methods}

\section{Detailed protocols}

Detailed bullet-point versions of the ACT2-seq and ACTseq protocols are available at The Protocol Exchange website (https://protocolexchange.researchsquare.com/).

\section{Reagents}

MinElute PCR Purification Kit (Qiagen catalog \# 28004). MinElute Gel Extraction Kit (Qiagen catalog \# 28604). Phusion 2X HF Master Mix (New England Biolabs catalog \# M0531S).

H3K4me3 antibody (Millipore Sigma catalog \# 07-473). H3K27ac antibody (Abcam catalog \# ab4729).

RNA Pol II antibody (Millipore Sigma catalog \# 17-620). Rabbit IgG isotype control (Abcam catalog \# ab37415).

\section{Public data sets}

The following publicly available data sets were used to validate the ACT2 results: ENCFF375WTP (H3K4me3), GSM3965505 (H3K27ac), ENCFF782FRS (H3K9me3), ENCFF865BUP (Pol II), and GSM4156601 (RNA-seq).

\section{Design and preparation of the PA-Tn 5 complex adapters}

As in ACT-seq, the oligonucleotide adapters that are bound by the PA-Tn5 enzyme are partially double stranded after being annealed to the pMENTS ("mosaicend, non-transfer strand") oligonucleotide. In ACT2, however, each adapter bears a distinct barcode sequence to enable the signals from each antibody to be distinguishable in the resulting sequence data. The general sequences are provided in the following table, given in $5^{\prime}$ to $3^{\prime}$ orientation:

\begin{tabular}{ll}
\hline Oligo Name & Sequence \\
\hline pMENTS & 5'Phos-CTGTCTCTTATACACATCT \\
Complex Adapter A & CCTACACGACGCTCTTCCGATCT \\
& NNNNNNNNAGATGTGTATAA \\
GAGACAG & TTCAGACGTGTGCTCTTCCGA \\
Complex Adapter B & TCTNNNNNNNAGATGTGTATAA \\
& GAGACAG \\
\hline
\end{tabular}

where the poly- $\mathrm{N}$ region represents the variable barcode sequences of 7 or 8 nucleotides in length. The sequences upstream (left, 5') of the barcodes are designed to anneal to either the i5 or i7 PCR primers that are used later in the protocol during library preparation.
The sequence downstream (right, $3^{\prime}$ ) of the barcodes is the mosaic end (ME) sequence that is bound by the Tn5 enzyme. We recommend varying the lengths of the barcodes between 7 and 8 bp to help promote sequence complexity in the resulting libraries. For a full list of all adapters used in this study, see Additional file 1.

Prior to use, each complex adapter was annealed to pMENTS using the following procedure: $25 \mu \mathrm{l}$ of $100 \mu \mathrm{M}$ complex adapter was mixed with $25 \mu \mathrm{l}$ of $100 \mu \mathrm{M}$ pMENTS. The mixture was heated to $99{ }^{\circ} \mathrm{C}$ for $5 \mathrm{~min}$, and the heat source was then turned off and the solutions were allowed to cool to room temperature over the course of two hours or overnight. The resulting $50 \mu \mathrm{M}$ annealed adapters were then stored at $4{ }^{\circ} \mathrm{C}$ or $-20^{\circ} \mathrm{C}$ until use.

\section{Design of the PCR library adapters}

The adapters used in the library preparation steps are distinct from the complex adapters detailed above. The library adapters are based on Illumina NextEra designs and are barcoded to enable different samples to be distinguished from one another after multiplexing. The general sequences are provided in the following table, given in $5^{\prime}$ to $3^{\prime}$ orientation:

\begin{tabular}{ll}
\hline Oligo name & Sequence \\
\hline Library Adapter A & AATGATACGGCGACCACCGAGATCT- \\
& ACACTCTTTCCCTACACGACGCTCTTCCGATCT \\
Library Adapter B & CAAGCAGAAGACGGCATACGAGAT- \\
& NNNNNNNNGTGACTGGAGTTCAGACGTGT \\
& GCTCTTCCGATCT
\end{tabular}

For this study, a single universal Library Adapter A sequence was used in all samples, whereas each sample received a variant of Library Adapter B containing a unique i7 index sequence (represented by the 8 bp poly$\mathrm{N}$ region in the table). This enables the samples to be distinguished from one another after multiplexing. For a full list of all adapters used in this study, see Additional file 1.

\section{Expression of recombinant PA-Tn5}

The expression vector containing the PA-Tn 5 construct is available from Addgene under accession number 121137 (http://www.addgene.org/121137/). Procedures for expression and purification of recombinant PA-Tn5 are provided in the original ACT-seq manuscript and in more detail in the bullet-point ACT2 protocol available on The Protocol Exchange.

\section{Preparation of the antibody-PA-Tn 5 complex}

Separate PA-Tn5 complexes with unique adapters were prepared for each antibody. Separate $1.5 \mathrm{~mL}$ tubes were labeled for each desired PA-Tn5 complex 
and the following was added: $4.5 \mu \mathrm{l}$ of $2 \mathrm{X}$ Complex Buffer $(0.1 \mathrm{M}$ Tris pH 8.0, 0.3 M NaCl, 0.1\% Triton $\mathrm{X}-100,25 \%$ glycerol) supplemented with an EDTAfree protease inhibitor cocktail to $2 \mathrm{X}$ concentration, $2 \mu \mathrm{l}$ of an annealed $50 \mu \mathrm{M}$ Complex Adapter A, $2 \mu \mathrm{l}$ of an annealed $50 \mu \mathrm{M}$ Complex Adapter B, and $2.5 \mu \mathrm{l}$ of $\sim 1 \mu \mathrm{g} / \mu \mathrm{l}$ purified recombinant PA-Tn 5 enzyme. The solutions were mixed by pipetting and incubated at room temperature for $10 \mathrm{~min}$.

A fresh set of $1.5 \mathrm{~mL}$ tubes was labeled for each biological sample. Each tube received $1.5 \mu \mathrm{l}$ of the appropriate complex prepared above, $1.5 \mu \mathrm{l}$ of $2 \mathrm{X}$ Complex Buffer, and $1.5 \mu \mathrm{l}$ of the matching antibody. The samples were mixed by pipetting and incubated at room temperature for $30 \mathrm{~min}$. The prepared antibody-PA-Tn 5 complexes can be left at room temperature prior to use for several hours if necessary. Note that we typically add the antibodies volumetrically due to the difficulty in obtaining accurate concentration measurements from many antibody manufacturers.

For probing multiple epigenetic marks in the same samples, separate aliquots of antibody-PA-Tn5 complexes should be prepared for the subsequent antibodies as well.

\section{Cell crosslinking and permeabilization}

Cells were crosslinked by incubating for $10 \mathrm{~min}$ in $10 \mathrm{ml}$ of culture medium supplemented with $0.25 \%$ formaldehyde. The cells were pelleted and washed twice in $10 \mathrm{ml}$ of PBS. 1 million cells were transferred to a $1.5 \mathrm{ml}$ tube and suspended in $1 \mathrm{ml}$ of freshly prepared RIPA Buffer $(150 \mathrm{mM} \mathrm{NaCl}, 0.2 \%$ SDS, $0.1 \%$ sodium deoxycholate, and $1 \%$ Triton X-100 in TE buffer $\mathrm{pH}$ 7.5) for $10 \mathrm{~min}$. The permeabilized cells were pelleted at $850^{*} \mathrm{~g}$ for $2 \mathrm{~min}$ and the supernatant was carefully removed to $\sim 50 \mu \mathrm{l}$. The cells were suspended in $1 \mathrm{ml}$ of Wash Buffer (50 mM Tris pH 8.0, $150 \mathrm{mM} \mathrm{NaCl}, 0.05 \%$ Triton X-100, 2 mM EDTA). The cells were pelleted as above, and the supernatant was removed to $\sim 50 \mu$ l. The cell suspension was diluted to $1 \mathrm{ml}$ using Wash Buffer to generate an approximate concentration of one thousand cells per microliter.

\section{Complex binding, washing, and tagmentation}

The cell suspension was gently mixed, and $50 \mu$ of this suspension was added to each of the sample tubes containing the first set of antibody-PA-Tn5 complexes. The samples were mixed gently and incubated for $30 \mathrm{~min}$ at room temperature to allow the complexes to bind to chromatin. After incubation, $1 \mathrm{ml}$ of Wash Buffer was added to each sample, and the tubes were sealed and mechanically rotated for $5 \mathrm{~min}$ at room temperature. The cells were pelleted at $850^{*} \mathrm{~g}$, and the supernatant was removed to $\sim 50 \mu \mathrm{l}$. At this point, $3 \mu \mathrm{l}$ of an annealed $50 \mu \mathrm{M}$ "blocking adapter" were added to each sample. The blocking adapter is any annealed Tn 5 complex adapter that features a barcode sequence that is not used in any of the samples. The samples were gently mixed and incubated at room temperature for 10 min to allow the blocking adapter to bind to any residual PA-Tn5 complexes that did not take up their original adapters.

After incubation with the blocking adapter, the second set of prepared antibody-PA-Tn 5 complex solutions were added to the matching samples. The samples were mixed gently and incubated at room temperature for $30 \mathrm{~min}$. $500 \mathrm{ul}$ of Wash Buffer was added to each sample, and the tubes were sealed and mechanically rotated for $5 \mathrm{~min}$ at room temperature. The cells were pelleted at $850^{\circ} \mathrm{g}$, and the supernatant was removed to $\sim 50 \mu \mathrm{l}$. The $500 \mu \mathrm{l}$ wash and pelleting steps were repeated once. Each sample was diluted to $100 \mathrm{ul}$ using Wash Buffer and received $1.5 \mu \mathrm{l}$ of $1 \mathrm{M} \mathrm{MgCl}_{2}$. The samples were mixed by pipetting and incubated at $37{ }^{\circ} \mathrm{C}$ for one hour to allow targeted tagmentation to occur.

\section{Purification of DNA fragments}

The tagmentation reactions were stopped by adding $8 \mu \mathrm{l}$ of $0.5 \mathrm{M}$ EDTA to each sample. The samples were mixed by vortexing and incubated at $80{ }^{\circ} \mathrm{C}$ for $5 \mathrm{~min}$ to deactivate the transposase. Each sample received $2 \mu \mathrm{l}$ of $10 \%$ SDS and $1 \mu \mathrm{l}$ of $20 \mathrm{mg} / \mathrm{ml}$ proteinase $\mathrm{K}$ enzyme. The samples were incubated at $55{ }^{\circ} \mathrm{C}$ for $60 \mathrm{~min}$. After the incubation, the samples were purified using a MinElute PCR Purification Kit and an elution volume of $20 \mu \mathrm{l}$. After purification, samples may be stored at $-20{ }^{\circ} \mathrm{C}$ until library preparation is performed.

\section{Library preparation}

The purified $\sim 20 \mu \mathrm{l}$ samples were transferred to PCR tubes for library preparation. Each tube received $0.5 \mu \mathrm{l}$ of $50 \mu \mathrm{M}$ universal Library Adapter A and $0.5 \mu \mathrm{l}$ of a $50 \mu \mathrm{M}$ Library Adapter B with a unique barcode. $20 \mu$ of $2 \mathrm{X}$ Phusion HF Master Mix was added to each sample and mixed. PCR was performed to amplify libraries using the following program:

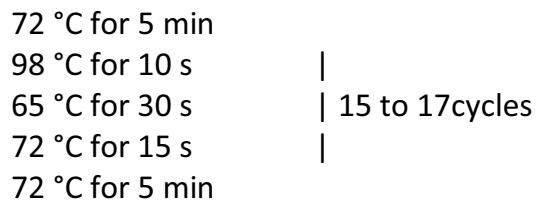

The resulting PCR products were visualized using gel electrophoresis on 2\% agarose E-gels. Gel slices were excised corresponding to DNA fragment sizes between $\sim 250$ and 850 bp. Gel purification was 
performed using a MinElute Gel Purification Kit. The purified DNA libraries were quantified using a Qubit fluorometer. Libraries were sequenced on Illumina's NovaSeq platform using PE150 format.

\section{Assembly of data sets from ACT2-seq libraries}

Each ACT2 sample comprised data from two or three concurrently profiled epigenetic marks along with cooccupancy data. Thus, the files required partitioning prior to analysis. For an example sample in which two factors were mapped using antibodies "A" and "B", five partitioned. fastq file pairs were generated for examination and analysis (Table 1, Additional file 3). These files were populated based on the combination of adapter barcode sequences at either end of the DNA fragments.

Reads from all sub-libraries were mapped separately to the human genome (hg19) using Bowtie2 [21]. Reads with a MAPQ quality score less than 10 and duplicate reads were removed. The specific sub-libraries used to generate each figure are noted in Additional file 2.

\section{Enriched regions and their annotations}

Enriched regions were identified using SICER [22] with the following parameters: window size of $200 \mathrm{bp}$, gap size of $200 \mathrm{bp}$, and an E-value of 100. Enriched regions were aligned to annotated genes using the intersect function from BEDTools [23]. Enhancer elements were defined as regions of $\mathrm{H} 3 \mathrm{~K} 27 \mathrm{ac}$ enrichment that were located at least five kilobases distant from an annotated TSS. The annotation of enriched genomic elements was performed using the annotatepeaks.pl utility of HOMER (http:// homer.ucsd.edu/homer/).

\section{Supplementary Information}

The online version contains supplementary material available at https://doi. org/10.1186/s13578-021-00711-4.

Additional file 1: Oligonucleotide adapters used in this study.

Additional file 2: Mapping statistics and sub-libraries used in each figure.

Additional file 3: Scripts used in data analysis and partitioning.

\section{Acknowledgements}

We thank the NHLBI DNA Sequencing Core Facility for sequencing the libraries.

\section{Authors' contributions}

BC and JP performed the experiments. BC, WLK, and KZ analyzed and interpreted the data. WLK performed the computational analysis of sequencing data. BC wrote the manuscript with substantial input from KZ and WLK. All authors read and approved the final manuscript.
Funding

Open Access funding provided by the National Institutes of Health $(\mathrm{NIH})$. The work was supported by Division of Intramural Research, National Heart, Lung and Blood Institute, NIH.

\section{Availability of data and materials}

All genome-wide data sets generated for this study are available at the Gene Expression Omnibus under Accession Number GSE179047.

\section{Declarations}

Ethics approval and consent to participate

Not applicable.

\section{Consent for publication}

Not applicable.

\section{Competing interests}

The authors declare that they have no competing interests.

Received: 29 September 2021 Accepted: 9 November 2021

Published online: 04 December 2021

\section{References}

1. Rothbart SB, Strahl BD. Interpreting the language of histone and DNA modifications. Biochim Biophys Acta. 2014;1839(8):627-43.

2. Soshnev AA, Josefowicz SZ, Allis CD. Greater than the sum of parts: complexity of the dynamic epigenome. Mol Cell. 2018;69(3):533.

3. Jambhekar A, Dhall A, Shi Y. Roles and regulation of histone methylation in animal development. Nat Rev Mol Cell Biol. 2019;20(10):625-41.

4. Li S, Yang D, Gao L, Wang Y, Peng Q. Epigenetic regulation and mechanobiology. Biophys Rep. 2020;6(2):33-48.

5. Gorkin DU, Leung D, Ren B. The 3D genome in transcriptional regulation and pluripotency. Cell Stem Cell. 2014;14(6):762-75.

6. Vermunt MW, Zhang D, Blobel GA. The interdependence of gene-regulatory elements and the 3D genome. J Cell Biol. 2019;218(1):12-26.

7. Rowley MJ, Corces VG. Organizational principles of $3 \mathrm{D}$ genome architecture. Nat Rev Genet. 2018;19(12):789-800.

8. Barski A, Cuddapah S, Cui K, Roh TY, Schones DE, Wang Z, et al. Highresolution profiling of histone methylations in the human genome. Cell. 2007;129(4):823-37.

9. Mikkelsen TS, Ku M, Jaffe DB, Issac B, Lieberman E, Giannoukos G, et al. Genome-wide maps of chromatin state in pluripotent and lineage-committed cells. Nature. 2007:448(7153):553-60.

10. Wang Z, Zang C, Rosenfeld JA, Schones DE, Barski A, Cuddapah S, et al. Combinatorial patterns of histone acetylations and methylations in the human genome. Nat Genet. 2008;40(7):897-903.

11. Bernstein BE, Mikkelsen TS, Xie X, Kamal M, Huebert DJ, Cuff J, et al. A bivalent chromatin structure marks key developmental genes in embryonic stem cells. Cell. 2006;125(2):315-26.

12. Carter B, Zhao K. The epigenetic basis of cellular heterogeneity. Nat Rev Genet. 2021;22(4):235-50.

13. Carter B, Ku WL, Kang JY, Hu G, Perrie J, Tang Q, et al. Mapping histone modifications in low cell number and single cells using antibody-guided chromatin tagmentation (ACT-seg). Nat Commun. 2019:10(1):3747.

14. Handa T, Harada A, Maehara K, Sato S, Nakao M, Goto N, et al. Chromatin integration labeling for mapping DNA-binding proteins and modifications with low input. Nat Protoc. 2020;15(10):3334-60.

15. Beischlag TV, Prefontaine GG, Hankinson O. ChIP-re-ChIP: co-occupancy analysis by sequential chromatin immunoprecipitation. Methods Mol Biol (Clifton, NJ). 2018;1689:103-12.

16. Tamura I, Ohkawa Y, Sato T, Suyama M, Jozaki K, Okada M, et al. Genomewide analysis of histone modifications in human endometrial stromal cells. Mol Endocrinol. 2014;28(10):1656-69. 
17. Hughes AL, Kelley JR, Klose RJ. Understanding the interplay between CpG island-associated gene promoters and H3K4 methylation. Biochim Biophys Acta Gene Regul Mech. 2020;1863(8):194567.

18. Papait R, Cattaneo P, Kunderfranco P, Greco C, Carullo P, Guffanti A, et al. Genome-wide analysis of histone marks identifying an epigenetic signature of promoters and enhancers underlying cardiac hypertrophy. Proc Natl Acad Sci USA. 2013;110(50):20164-9.

19. Ma S, Zhang B, LaFave LM, Earl AS, Chiang Z, Hu Y, et al. Chromatin potential identified by shared single-cell profiling of RNA and chromatin. Cell. 2020;183(4):1103-16.e20.

20. Deaton AM, Bird A. CpG islands and the regulation of transcription. Genes Dev. 2011;25(10):1010-22.

21. Langmead B, Salzberg SL. Fast gapped-read alignment with Bowtie 2. Nat Methods. 2012;9(4):357-9.
22. Xu S, Grullon S, Ge K, Peng W. Spatial clustering for identification of ChIP-enriched regions (SICER) to map regions of histone methylation patterns in embryonic stem cells. Methods Mol Biol (Clifton, NJ). 2014;1150:97-111.

23. Quinlan AR, Hall IM. BEDTools: a flexible suite of utilities for comparing genomic features. Bioinformatics. 2010;26(6):841-2.

\section{Publisher's Note}

Springer Nature remains neutral with regard to jurisdictional claims in published maps and institutional affiliations.
Ready to submit your research? Choose BMC and benefit from:

- fast, convenient online submission

- thorough peer review by experienced researchers in your field

- rapid publication on acceptance

- support for research data, including large and complex data types

- gold Open Access which fosters wider collaboration and increased citations

- maximum visibility for your research: over 100M website views per year

At BMC, research is always in progress.

Learn more biomedcentral.com/submissions 